\title{
Estimates of demographic parameters for southern right whales (Eubalaena australis) observed off Península Valdés, Argentina
}

\author{
J.G. CoOKE*, V.J. RowntreE ${ }^{\dagger}$ AND R. PAYNE \\ Contacte-mail:jgc@cems.de
}

\begin{abstract}
Photographs of the right whales which occur between June and December in the waters surrounding Península Valdés, Argentina, have been obtained from aerial surveys conducted each year from 1971 to the present. Resightings of previously catalogued individuals enable various demographic parameters to be estimated. From analyses of multiple resightings of females accompanied by calves, estimates of the following demographic parameters were obtained, based on the data collected during 1971-90: mean calving interval 3.35 yrs $(\mathrm{SE}=0.05$ $\mathrm{yrs})$; mean age at first calving $9.1 \mathrm{yr}(\mathrm{SE}=0.3 \mathrm{yr})$; adult female annual mortality rate $0.019(\mathrm{SE}=0.005)$; annual percentage rate of population increase $6.9 \%(\mathrm{SE}=0.7 \%)$; reproductive female population size in 1990: 328 animals $(\mathrm{SE}=21)$. No evidence of any trend with time in mean calving interval, mortality rate or rate of population increase was found.
\end{abstract}

KEYWORDS: SOUTHERN RIGHT WHALES; PHOTO-IDENTIFICATION; INDIVIDUAL RECOGNITION; BIOLOGICAL PARAMETERS

\section{INTRODUCTION}

Photo-identifications of individual whales based on natural markings have been obtained from aerial surveys each year since 1971 to study a population of southern right whales (Eubalaena australis) on their winter assembly grounds around Península Valdés, Argentina. The methods used to record natural markings and to identify resightings of individual whales, and the characteristics of the study season and area, are described by Payne (1986) and Payne et al. (1983).

An analysis of the data to estimate population size and demographic parameters was conducted by Payne et al. (1990) using data from 1971-1986. For the present analysis, the data for the period 1971-1990 were available. The study is ongoing and photographs have continued to be taken each year, however, photographs after 1990 have not yet been catalogued.

A total of 1,223 distinct individuals was catalogued up to 1990, and each individual was seen in an average of 2.4 different years. Some 339 individually identified whales were observed at least once in definite association with a calf, and each of these were recorded in an average of 2.15 different years with a calf, giving a total of 728 observed calvings, where an 'observed calving' means that the mother was individually identified and recorded as having a calf, even if the calf was not individually photo-identifiable (Fig. 1).

Survey effort varied from year to year, and therefore the numbers identified in each year do not necessarily reflect the numbers of whales present.

For the reasons discussed by Payne et al. (1990), only the sightings of females accompanied by calves are used to estimate population parameters. The reasons for not using the other components include:

(1) animals not previously seen with a calf are of unknown sex and maturity status, whereas the probability of a whale entering the study area during the study period may well depend on sex and maturity status;
(2) although some females were seen in the study area in years in which they apparently had no calf, the absence of an accompanying calf in an observation does not necessarily imply that no calf is present, hence it is not possible to divide observations of females into those with and without calves.

Conventional mark release models are not suitable for these data because of the periodic nature of the calving process. Breeding females calve at intervals of 2-5 years, mainly 3 years (Fig. 2). Although females are sometimes observed in the study area in years in which they do not calve, the probability of them being in the area and being observed appears to be less than in years in which they calve. Of whales which had been previously observed to calve, there were a total of 389 resightings with calves, but only 242 resightings without calves. Since the average calving interval is at least three years (see below), these figures imply that a whale is much more likely to be seen in the study area when it has a calf than when it does not. Hence, the assumptions of independence of sightings probabilities from year to year that are required for most mark release models are not fulfilled.

The birth interval model of Barlow and Clapham (1997) is also not applicable to these data, since it relies on the assumption that the probability of sighting a whale is independent of whether or not it has a calf.

The distribution of observed calving intervals (Fig. 3) does not directly reflect the true calving interval distribution because not all calvings are observed. Not all calvings that occur in the study area are necessarily recorded because not all whales in the study area are recorded each year and not all calves have yet been born when the mother is sighted. The calf, even if present, is not always seen when the mother is sighted, nor is it always in sufficiently close or unique association with an adult for the latter to be definitely identified as the parent (for example when there are two or more adults in close proximity to the calf). Furthermore, some calvings in the population may occur outside the study area. Two females that had been recorded with calves off

\footnotetext{
* Centre for Ecosystem Management Studies, Mooshof, 79297, Germany.

${ }^{\dagger}$ Whale Conservation Institute, University of Utah, Department of Biology, 256 South 1400 East, Salt Lake City, UT, 84112, USA.

* Whale Conservation Institute, 191 Weston Road, Lincoln, MA 01773, USA.
} 


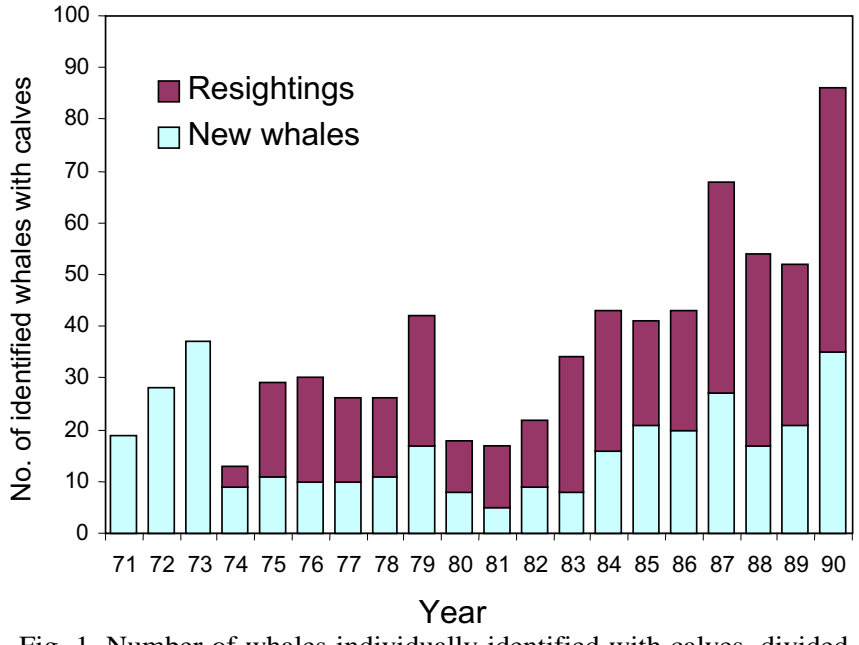

Fig. 1. Number of whales individually identified with calves, divided into those observed to calve for the first time, and those observed to calve for a second or subsequent time.

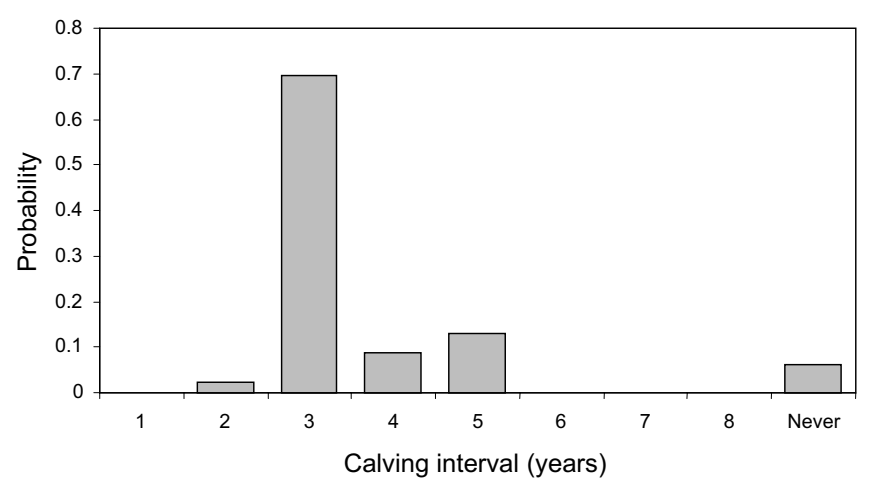

Fig. 2. Estimated distribution of calving intervals in the breeding population.

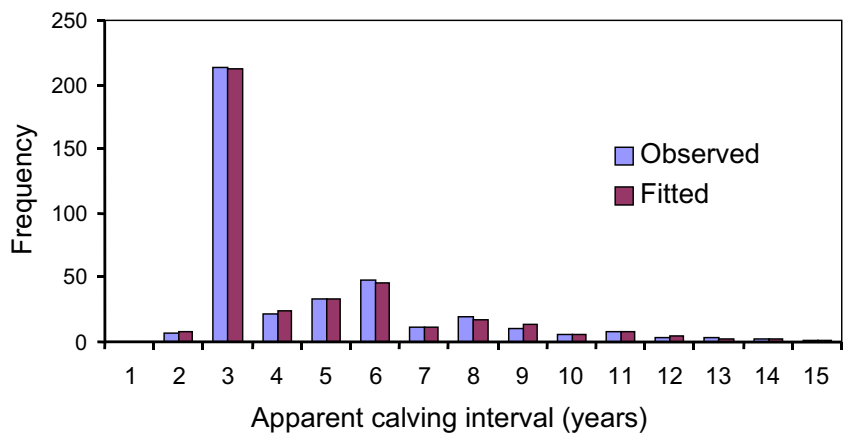

Fig. 3. Observed and fitted distributions of intervals between observed calvings of individually identified whales.

Península Valdés were resighted with calves in later years in a different nursery area off Laguna, southern Brazil (Best et al., 1993).

In this paper an amended version of the model developed by Payne et al. (1990) is applied to the 1971-90 data to estimate the pattern of calving intervals and other demographic parameters.

\section{MATERIAL AND METHODS}

As noted above, the observation of a female in a given year without a calf does not necessarily imply that it did not calve in that year. Hence, only positive identifications of females with calves are used in this analysis. A female is recorded as

calving in a given year if it is seen at least once in exclusive close association with a calf in that year, using the criteria given by Payne and Dorsey (1983).

The data consist of sequences of observed calvings of whales. There is one sequence for each of the 339 whales that was observed to calve at least once in the study period (1971-90 for this analysis). Each sequence is of the form: $\left(t_{i j}\right.$, $j=1, \ldots, k_{i}$ ) where $t_{i j}$ is the year of the $j$ th observed calving of whale $i$ and $k_{i}$ is the number of observed calvings of whale $i$. For example, a typical sequence might be: (1973, 1976, 1983, 1989).

Although relatively few calves were observed with distinctive markings that enabled them to be re-identified subsequently as adults, those few provide potentially valuable information on the age at first calving. Of 246 calves entered into the catalogue, 25 were subsequently re-identified with calves of their own.

We first outline a method for interpreting the calving sequence data and then develop an extension to make use of the identifications of whales as calves. To interpret the calving sequence data, two models are required:

(1) a model of the population which includes a model of the calving sequences occurring in the population;

(2) a model of the sampling process which enables determination of the likelihood or goodness-of-fit of the population model to the observed data.

The parameters of the population model are estimated by finding the parameter values that maximise the likelihood of the observed data.

\section{Population model}

The model is of the female breeding population. An individual is considered a member of the breeding population from the year of its first calf to the year of its last calf, both years inclusive.

\section{Generic population model}

We first define a generic model which involves minimal assumptions but many potential parameters, and then consider special cases of the generic model involving fewer unknown parameters. The most parsimonious model giving acceptable fit to the data will be selected.

The model contains the following parameters:

$C_{t}$

$R_{t}$

$N_{t}$

$m$

$h_{t, i}(i=1, \ldots, m)$ probability that a whale calving in year $t$

will next calve in year $t+i(i=1, \ldots, m)$.
The probability that a whale calving in

will next calve in year $t+i(i=1, \ldots, m)$.
The probability that a whale calving in year $t$ never calves again is $1-\sum_{i} h_{t, i}$.

expected number of calvings in year $t$. It is convenient to define $t$ so that $t=0$ refers to the first year for which there are data. The actual number of calvings in year $t$ is assumed to have a Poisson distribution with expectation $C_{t}$. expected recruitment to the breeding population in year $t$ (expected number of animals calving in year $t$ for the first time). The actual number of first calvings in year $t$ is assumed to have a Poisson distribution with expectation $R_{t}$. expected size of the female breeding population in year $t$. maximum interval between successive calvings of an individual. 
As will become apparent, it is necessary to model the population parameters from $m$ years prior to the start of the data series, up until the end of the data series $(t=-m, \ldots, T$, where $T$ is the last year with data).

The $C_{t}$ satisfy the recurrence relation:

$$
C_{t}=R_{t}+\sum_{i=1}^{m} h_{t-i, i} C_{t-i}
$$

The expected breeding population in year $t, N_{t}$, is given by:

$$
N_{t}=C_{t}+\sum_{i=1}^{m-1} C_{t-i} \sum_{j=i+1}^{m} h_{t-i, j}
$$

The first term on the right hand side of this expression is the expected number of individuals calving in year $t$; the second term is the expected number of individuals which do not calve in year $t$ but which have two adjacent calvings that straddle year $t$. The expression reflects the definition of the breeding population as being females between the year of their first calf and the year of their last calf, both end points inclusive.

Mortality is not featured explicitly in the model. It is implicitly included in the probability that a whale will never calve in the study area again, if 'mortality' is defined to include reproductive senescence and/or permanent emigration as well as actual death.

\section{ASSUMPTIONS}

The probability that a whale calving in year $t$ will next calve in year $t+i$ is assumed to be independent of its calving history prior to year $t$. Under this assumption, the parameters $h_{t, t+i}$ determine the calving sequence process in the breeding population.

\section{Minimal population model}

The minimal model is the simplest meaningful special case of the generic model. The minimal model includes the following additional assumptions:

(a) the calving interval probabilities are constant over time: $h_{t, i}=h_{i}$

(b) the expected recruitment (number of first calvings) increases exponentially at a constant annual rate, $r$ :

$$
R_{t+1}=R_{t}(1+r)
$$

Under these assumptions, the expected number of calvings, $C_{t}$, and the breeding population size, $N_{t}$, also increase at the same annual rate, $r$.

The minimal model contains $(m+2)$ independent biological parameters to be estimated: $h_{i}(i=1, \ldots, m), r$, and $C_{0}$, the expected number of calvings in the initial year. When the values of these parameters are specified, the full sequence of $C_{t}(t=-m, \ldots, T)$ and $N_{t}$ can be derived using equations (1) and (2). The procedure for determining the choice of $m$, the maximum calving interval, is described below.

For computational purposes it is convenient to work with the following transformation of the $h_{i t}$ :

$$
\lambda_{i t}=\log \left(h_{i t} /\left(1-\sum_{j=1}^{i} h_{j t}\right)\right)
$$

There is a 1:1 relationship between the $\lambda_{i t}$ and the $h_{i t}$, but each of the $\lambda_{i t}$ can take any value in the range $(-\infty,+\infty)$. Some derived parameters of interest are defined in the following sections.

\section{Implied survival/mortality rates}

The equivalent annual survival rate, $S$, corresponding to a given set of calving interval probabilities, $h_{i}$, is the level of annual survival that would give the specified probability of a given calving being the last calving if the survival probability were independent of the calving history. $S$ is the value which satisfies the following equation:

$$
\sum_{i=1}^{m} h_{i} S^{-i}=1
$$

If survival rate is affected by calving history, then the equivalent survival rate is a form of weighted average of the history-specific rates. The equivalent instantaneous mortality rate is given by:

$$
M=\ln S
$$

The quantities, $h_{i}^{*}=h_{i} S^{-i}$, can be regarded as conditional calving interval probabilities, conditional on survival to the next calving.

\section{Mean calving interval}

The mean calving interval is given by:

$$
\bar{h}=\sum_{i=1}^{m} i h_{i} / \sum_{i=1}^{m} h_{i}
$$

\section{Mean calving rate}

We define the 'mean' calving rate to be the ratio of the expected number of calves produced in a year to the expected breeding population size in that year: $C_{t} / N_{t}$. Because we have defined an individual's membership of the breeding population to begin and end with a calving, our definition of calving rate may yield slightly higher values than some other definitions in use.

\section{Deviations from the minimal model}

After fitting the minimal model, we investigated whether an improved fit to the data could be obtained by allowing additional parameters. The following extensions to the minimal model were considered, each involving one extra parameter.

(1) time trend in mortality rate: $M_{t}=M_{0}+t \mu(\mu$ constant);

(2) time trend in rate of increase: $r_{t}=r_{0}+t \rho$ ( $\rho$ constant);

(3) time trend in calving interval probabilities: $\lambda_{t, i}=\lambda_{i}+\gamma t$ ( $\gamma$ constant $)$.

Positive values of $\gamma$ correspond to a tendency for calving intervals to decrease with time.

When investigating a possible time trend in mortality rate, the conditional calving interval probabilities, $h_{i}$, are assumed constant over time. When looking for a time trend in the calving interval probabilities, these are rescaled so that the equivalent mortality rate remains constant over time. Initially, one is looking only for evidence of specific directions of departure from the minimal model. Only when such evidence is found is it worthwhile considering in more detail the nature of such departures.

\section{Modelling the sampling process}

For the model of the sampling process, the following additional notation is required: 
$n_{t} \quad$ number of distinct calvings observed in year $t$;

$n_{t, i}$ number of individuals that were observed to calve in year $t$ and were next observed to calve in year $t+i$ $(i>0)$;

$n_{t, O} \quad$ number of individuals that were observed to calve in year $t$ but not subsequently;

$n_{-, t}$ number of individuals that were first observed to calve in year $t$;

$T \quad$ last year of data ( $T=0$ is the first year of data, hence there are $T+1$ years of data in total unless some years were missed);

$U_{t}$ expected number of calvings (not necessarily observed) in year $t$ by individuals who have not previously been observed to calve;

$p_{t} \quad$ probability that a calving occurring in year $t$ will be observed: $p_{t}=0$ for $t<0$;

$g_{t, i}$ probability that an individual that calves (but is not necessarily observed) in year $t$ will next be observed to calve $i$ years later $(i>0)$;

$g_{t, 0}$ probability that an individual that calves (but is not necessarily observed) in year $t$ will not be observed to calve again within the period of the current dataset.

\section{ASSUMPTIONS}

The probability that a random calving in a given year will be recorded is assumed to be independent of its previous calving record. Under this assumption, the expected frequency of a calving sequence $\left(t_{1}, t_{2}, \ldots\right)$ is the product of the expected frequency of the initial observation in the sequence (the expected number of first observed calvings in year $t_{1}$ ) times the product of the probabilities of each subsequent step in the sequence given the sequence to date.

Following Cormack (1981), the frequencies of each possible calving sequence are treated as independent random variables each with a Poisson distribution. To evaluate the likelihood of the data, we require an expression for the expected frequency of a given calving sequence. $C_{t}$ :

The following equations yield the values of $U_{t}$ in terms of

$$
\begin{gathered}
U_{t}=C_{t}(t \leq 0) \\
U_{t}=R_{t}+\sum_{i=1}^{m} h_{t-i, t} U_{t-i}\left(1-p_{t-i}\right)(t>0)
\end{gathered}
$$

The expected number of whales first observed to calve in year $t$ is $p_{t} U_{t}$.

The probability that a whale observed to calve in year $t$ is next observed to calve in year $t+i$ is given by:

$$
\begin{aligned}
& g_{t, l}=h_{t, 1} p_{t+1} \\
& g_{t, i}=h_{t, i} p_{t+i}+\sum_{j=1}^{i-1} h_{t, j}\left(1-p_{t+j}\right) g_{t+j, i-j} \quad(i=2, \ldots, m) \\
& g_{t, i}=\sum_{j=1}^{m} h_{t, j}\left(1-p_{t+j}\right) g_{t+j, i-j} \quad(i>m)
\end{aligned}
$$

The probabilities $g_{t, i}$ are calculated recursively: first for $i=1$, then for $i=2$, and so on.

The probability of the terminal step in a sequence (the probability that a whale observed to calve in year $t$ is not observed to calve again) is given by:

$$
\begin{aligned}
& g_{t, 0}=1-\sum_{i=1}^{T-t} g_{1, i} \quad(t=0, \ldots, T-1) \\
& g_{T, 0}=1
\end{aligned}
$$

The expected frequency of a given observed calving sequence $\left(t_{1}, t_{2}, \ldots, t_{n}\right)$ is thus:

$$
p_{t} U_{i}\left(\prod_{j=1}^{n-1} g_{t_{j},{ }_{j+1}}\right) g_{t_{n}, 0}
$$

Hence, the log-likelihood of the entire dataset, ignoring constant terms, is given by:

$$
L=\sum_{t=0}^{T}\left[n_{-, t} \ln \left(p_{t} U_{t}\right)-p_{t} U_{t}+\sum_{i=0}^{\min (m, T-t)} n_{t, i} \ln g_{t, i}\right]
$$

The parameters are estimated by maximising the log-likelihood over the free parameters. There are $(m+2)+$ $(T+1)$ free parameters to estimate in the minimal model: $C_{0}$, $r, h_{i}(i=1, \ldots, m)$, and $p_{t}(t=0, \ldots, T)$. In each of the three alternative models listed above there is one extra parameter. The values of all other parameters are calculated from these basic parameters using the above equations.

The expression $n_{t} / p_{t}$ provides an estimator for the actual (as opposed to the expected) number of calvings occurring in year $t$.

The variances of each parameter estimate are estimated from the curvature of the log-likelihood. Using vector notation, let $\mathbf{x}$ be the vector of free parameters and $\mathbf{z}$ be the vector of calculated parameters. Then:

$$
\begin{gathered}
\operatorname{var}(\mathbf{x}) \cong\left(\delta^{2} L / \delta x^{2}\right)^{-1} \\
\operatorname{var}(\mathbf{z}) \cong(\delta z / \delta x) \operatorname{var}(\mathbf{x})(\delta z / \delta x)^{\mathrm{T}}
\end{gathered}
$$

where the superscript ${ }^{\mathrm{T}}$ denotes transpose.

There remains the question of how to choose the value of $m$, the maximum length of calving interval. Because a model with a low value of $m$ is a special case of a model with a higher value of $m$, the likelihood increases with increasing $m$. We therefore choose, as a conservative estimate of $m$, the lowest value of $m$ that is consistent with the data in the sense of not being rejected in favour of a higher value of $m$ in a likelihood ratio test. This is not necessarily the best criterion for general use, but, as noted below, the results in this case indicate $m=5$ so clearly that a detailed consideration of the selection criteria for $m$ is not necessary for this particular dataset.

\section{Age at first calving}

Some of the calves observed had distinctive markings that enabled them to be re-identified subsequently as adults. Since these animals are of known age, they provide information on the age at first reproduction. The distribution of ages at first observed calving of known-age animals is shown in Fig. 4. These do not necessarily correspond to the true age at first calving because not all calvings are seen. Although the sample of known-age animals that have themselves been seen to calve is still small, a method of analysis is outlined here with a view to re-applying it when the data for more years become available.

The true distribution of age at first calving can be investigated by extending the above model to include the observations of identified individuals as calves. The following additional parameters are required:

$$
l
$$

$f_{t, i}(i=1, \ldots, l) \quad$ probability that a calf in year $t$ will first calve itself at age $i$; 
probability that a calf in the catalogue has sufficiently distinctive marks to be re-identifiable as an adult;

$q_{t, i}$ probability that a calf observed with distinctive markings in its year of birth $t$ will be first observed to calve at age $i$ $(i=1, \ldots, l)$; distinctive markings in year $t$ will not be observed to calve again within the period of data.

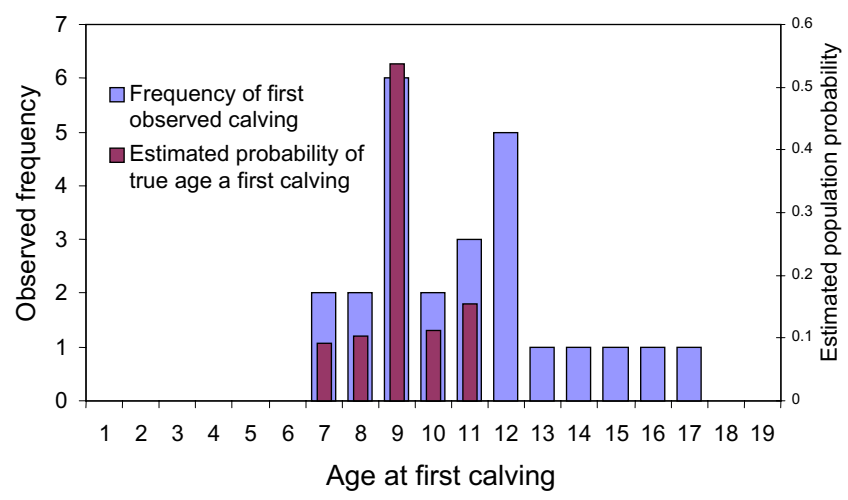

Fig. 4. Distribution of age at first observed calving of known-age individuals, and estimated probability distribution of true age at first calving in the population.

The following additional data are used:

$a_{t, i}(i=1, \ldots, l)$ number of calves observed with distinct markings in year $t$ which are subsequently first observed to calve at age $i$;

$a_{t, 0} \quad$ number of calves observed with distinct markings in year $t$ which have not subsequently been observed to calve.

The $q_{t, i}$ are given by the following expressions:

$$
\begin{array}{r}
q_{t, i}=\alpha\left[f_{t, i} p_{t+i}+\sum_{j=1}^{i-1} f_{t, j}\left(1-p_{t+j}\right) g_{t+j, i-j}\right] \quad \begin{array}{r}
(i=1, \ldots, l) \\
q_{t, i}=\alpha\left[\sum_{j=1}^{l} f_{t, j}\left(1-p_{t+j}\right) g_{t+, i-j}\right] \quad(i>l) \\
q_{t, 0}=1-\sum_{j=1}^{T-t} q_{t, j}
\end{array}
\end{array}
$$

For each $t$, the $f_{t, i}$ will sum to less than unity; many calves will never themselves have a calf, for example because they are male or die before attaining maturity. The parameter $\alpha$ is a nuisance parameter, which can be discarded and subsumed into the $f_{t, i}$, if the latter are interpreted as relative rather than absolute proportions.

The mean age at first calving for females born in year $t$ is given by:

$$
\sum_{i=1}^{l} i_{t, i} / \sum_{i=1}^{l} f_{t, i}
$$

The age at which a whale identified as a calf is first observed to have its own calf can be treated as a multinomial random variable, taking values $\{0, \ldots, l\}$ with probabilities $q_{t, j}(j=0$,

..., l) where the value zero means 'never or not yet'. Hence, the log-likelihood of these data, ignoring constant terms, is given by:

$$
L_{2}=\sum_{t=0}^{T-1} \sum_{j=0}^{\min (l, T-t)} a_{t, j} \ln q_{t, j}
$$

This log-likelihood is added to the calving-sequence log-likelihood given in expression (10), to yield a total log-likelihood. Since the sample size of known-age animals is currently too small to permit examination of possible changes in the age at first calving over time, we assume for this analysis that the $f_{t, i}$ are time-invariant, i.e. $f_{t, i}=f_{i}$. The $f_{i}$ are estimated along with the parameters of the calving-sequence model by maximising the total log-likelihood.

The maximum age at first calving, $l$, is chosen by finding the minimum value of $l$ which is consistent with the data in terms of not being rejected in favour of a higher value of $l$. This procedure yields a conservative estimate of the mean age at first calving.

\section{Survival rate of calves to first reproduction}

Since not all calves have sufficiently distinctive marks to be re-identifiable as adults, a direct estimate of survival from birth to maturity based on the proportion of calves subsequently identified as adults would be liable to be negatively biased. We can, however, estimate the survival rate of calves to first reproduction that would be needed to yield the estimated recruitment to the breeding population, on the assumption that all recruits were born within the population.

Assuming a 50:50 sex ratio at birth, the required survival probability of female calves to first reproduction is given by:

$$
S_{t}^{*}=2 R_{t} / \sum_{i=1}^{l} C_{t-i} f_{i}
$$

This quantity is time-invariant for the minimal model. Strictly speaking, it represents the probability that an observed female calf will survive to produce a calf: mortality between birth and the time of the initial observation of the calf is not included.

\section{RESULTS}

\section{Calving intervals}

Values less than five years for the maximum calving interval $(m)$ are clearly rejected on a likelihood test $(P \approx 0)$, whereas values of 5, 6, 7 or 8 fit the data virtually equally well (Table $1)$. This is reflected both in the virtually constant likelihood for $m \geq 5$, and in the estimated probabilities of 6-8 year intervals being close to zero. Thus, the data are consistent with a maximum interval of five years, and indicate that longer intervals, if they do occur, are rare in the population.

The fit between the observed and predicted distributions of observed calving intervals from the model with $m=5$ is clearly acceptable $\left(\chi^{2}=2.5\right.$, d.f. $=11, P>0.5$; Fig. 3$)$. Hence the value $m=5$ is selected for all the results that follow. The model adequately explains all observed calving intervals of six years or more as combinations of shorter intervals with one or more intermediate missed calvings. Because intervals shorter than three years are rare, virtually all observed intervals up to and including five years will be genuine calving intervals, without intervening missed calvings. 
Table 1

Selection of the maximum calving interval.

\begin{tabular}{|c|c|c|c|c|c|c|}
\hline \multirow[b]{3}{*}{ Interval } & \multicolumn{6}{|c|}{ Maximum calving interval $(\mathrm{yr})$ in model } \\
\hline & 3 & 4 & 5 & 6 & 7 & 8 \\
\hline & \multicolumn{6}{|c|}{ Estimated interval probabilities } \\
\hline 1 & 0.000 & 0.000 & 0.000 & 0.000 & 0.000 & 0.000 \\
\hline 2 & 0.215 & 0.110 & 0.023 & 0.023 & 0.022 & 0.021 \\
\hline 3 & 0.741 & 0.751 & 0.692 & 0.692 & 0.689 & 0.649 \\
\hline 4 & & 0.089 & 0.089 & 0.089 & 0.084 & 0.079 \\
\hline 5 & & & 0.131 & 0.131 & 0.131 & 0.118 \\
\hline 6 & & & & 0.000 & 0.000 & 0.025 \\
\hline 7 & & & & & 0.008 & 0.015 \\
\hline 8 & & & & & & 0.022 \\
\hline Never & 0.045 & 0.050 & 0.065 & 0.065 & 0.066 & 0.070 \\
\hline Mean interval & 2.774 & 2.977 & 3.351 & 3.351 & 3.381 & 3.584 \\
\hline Log-likelihood & -920.3 & -886.1 & -862.1 & -862.1 & -862.0 & -861.9 \\
\hline verdict & reject & reject & select & & & \\
\hline
\end{tabular}

The estimated distribution of calving interval probabilities is bimodal, with three years as the most common interval, but with five-year intervals more common than four-year intervals (Fig. 2). To assess whether the bimodal pattern was a significant result or merely a chance effect, the likelihood was re-calculated with the probabilities of four- and five-year intervals interchanged. The deterioration in goodness of fit was significant $(P<0.01)$, which suggests that the observed bimodal pattern is unlikely to be due to chance.

We conjecture that five-year intervals are more common than four-year intervals because they are caused by a specific event, such as a pre- or perinatal loss of a calf around the end of the usual three year interval, followed by a reduced interval of two rather than the usual three years to the next birth because of the skipped lactation.

\section{Population parameters and trends}

Precise estimates were obtained of the annual mortality rate in the breeding population, the mean calving interval, the mean calving rate, the $R_{\mathrm{II}}$ recruitment rate, and the annual rate of population increase (Table 2). The increase in the population is highly significant (estimated annual rate $6.9 \%$, $\mathrm{SE}=0.7 \%$ ).

Table 2

Estimates of various demographic parameters.

\begin{tabular}{|c|c|c|c|}
\hline \multicolumn{2}{|l|}{ Parameter } & Estimate & S.E. \\
\hline \multicolumn{2}{|l|}{ Annual rate of increase } & 0.069 & 0.007 \\
\hline \multicolumn{2}{|l|}{ Equivalent annual mortality rate } & 0.019 & 0.005 \\
\hline \multicolumn{2}{|l|}{ Equivalent annual survival rate } & 0.981 & 0.005 \\
\hline \multicolumn{2}{|l|}{ Mean calving interval } & 3.35 & 0.05 \\
\hline \multicolumn{2}{|l|}{ Mean calving rate } & 0.338 & 0.005 \\
\hline \multirow[t]{5}{*}{ Probability of interval length } & 1 year & 0.000 & \\
\hline & 2 years & 0.023 & 0.009 \\
\hline & 3 years & 0.695 & 0.023 \\
\hline & 4 years & 0.089 & 0.014 \\
\hline & 5 years & 0.131 & 0.019 \\
\hline \multicolumn{2}{|l|}{$\mathrm{R}_{\mid l}$ recruitment rate $\left(\mathrm{R}_{1} / \mathrm{N}_{1}\right)$} & 0.085 & 0.007 \\
\hline \multicolumn{2}{|c|}{ Mean age at first calving } & 9.1 & 0.3 \\
\hline \multicolumn{2}{|c|}{ Mean survival to first reproduction } & 0.92 & 0.11 \\
\hline
\end{tabular}

The breeding population is estimated to have increased from 92 individuals $(\mathrm{SE}=9)$ in 1971 to 328 in 1990 $(\mathrm{SE}=21) \quad($ Table 3$)$. The estimated annual sampling probability ranged from a low of 0.29 in 1981 to a high of 0.90 in 1973 (Table 3). Since the survey effort varied substantially from year to year, the variation in sampling probability does not necessarily reflect any variation in the presence of whales in the study area.

Table 3

Estimated numbers of calvings, female breeding population, and sampling probability by year.

\begin{tabular}{lcccccc}
\hline & & & & & Sampling \\
Year & Calvings & S.E. & Population & S.E. & probability & S.E. \\
\hline 1971 & 30 & 3 & 92 & 9 & 0.63 & 0.05 \\
1972 & 35 & 2 & 98 & 9 & 0.80 & 0.04 \\
1973 & 41 & 1 & 105 & 9 & 0.90 & 0.02 \\
1974 & 37 & 3 & 112 & 9 & 0.35 & 0.03 \\
1975 & 41 & 2 & 120 & 9 & 0.71 & 0.04 \\
1976 & 44 & 3 & 128 & 9 & 0.69 & 0.04 \\
1977 & 46 & 3 & 137 & 9 & 0.56 & 0.03 \\
1978 & 50 & 3 & 147 & 9 & 0.52 & 0.03 \\
1979 & 54 & 2 & 157 & 9 & 0.78 & 0.03 \\
1980 & 56 & 3 & 168 & 9 & 0.32 & 0.02 \\
1981 & 60 & 3 & 179 & 9 & 0.29 & 0.01 \\
1982 & 65 & 3 & 192 & 10 & 0.34 & 0.01 \\
1983 & 69 & 3 & 205 & 10 & 0.49 & 0.02 \\
1984 & 74 & 3 & 219 & 11 & 0.58 & 0.03 \\
1985 & 79 & 4 & 234 & 12 & 0.52 & 0.02 \\
1986 & 85 & 4 & 251 & 13 & 0.51 & 0.02 \\
1987 & 91 & 4 & 268 & 15 & 0.75 & 0.04 \\
1988 & 97 & 5 & 287 & 16 & 0.56 & 0.03 \\
1989 & 104 & 6 & 306 & 19 & 0.50 & 0.03 \\
1990 & 111 & 7 & 328 & 21 & 0.77 & 0.05 \\
\hline
\end{tabular}

There is some indication of a three-year cyclic fluctuation in the estimated annual numbers of calvings, relative to the expected numbers, which is not predicted by the model (Fig. $5)$. The initial variation in numbers of calvings by year is predicted by the model to even out over time because the average calving interval is not exactly three years. The mechanism which causes such fluctuations to persist merits further investigation.

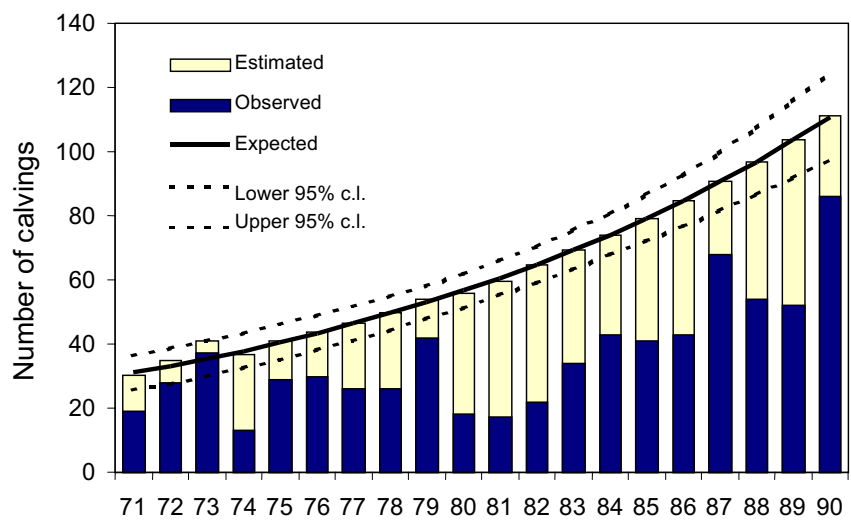

Fig. 5. Observed and estimated numbers of calvings by year, and expected numbers (fitted exponential trend) with $95 \%$ confidence limits.

Deviations from the minimal model and additional diagnostics

None of the three one-parameter extensions to the minimal model yielded a significant improvement in fit $(P>0.1$; Table 4). We conclude that there is as yet no evidence for any 
change over time in the mean calving interval, the adult mortality rate or the rate of population increase. These conclusions should be re-examined when the data for a longer series of years have become available.

Table 4

Tests for extensions to the minimal population model.

\begin{tabular}{lcccc}
\hline Nature of extension to model & Estimate & S.E. & $\Delta L^{\prime}$ & Significance \\
\hline Annual trend in mortality rate & 0.0022 & 0.0016 & 0.9 & $P>0.1$ \\
Annual trend in rate of increase & 0.00038 & 0.0035 & 0.6 & $P>0.1$ \\
Annual trend in mean calving & -0.0047 & 0.0042 & 0.8 & $P>0.1$ \\
interval & & & &
\end{tabular}

Increase in log-likelihood when parameter is included in model.

\section{Age at first calving}

The minimum observed age at first calving is seven years (Fig. 4). Models with maximum ages of first calving of eight years or less are clearly rejected $(P \approx 0$; Table 5$)$. On a one-tailed test, which seems appropriate in this case, a maximum age at first calving of 9 years is just rejected in favour of 10 years, which is itself just rejected in favour of 11 years $(P<0.05$ in each case $)$. The value of 11 years is not rejected in favour of higher values $(P>0.1)$.

Table 5

Selection of the maximum age at first calving.

\begin{tabular}{|c|c|c|c|c|c|c|}
\hline \multirow{3}{*}{$\begin{array}{l}\text { Age at first } \\
\text { calving }\end{array}$} & \multicolumn{6}{|c|}{ Maximum age at first calving (yr) in model } \\
\hline & 7 & 8 & 9 & 10 & 11 & 12 \\
\hline & \multicolumn{6}{|c|}{.................................. Estimated probabilities } \\
\hline 7 & 1.00 & 0.69 & 0.18 & 0.10 & 0.09 & 0.09 \\
\hline 8 & & 0.31 & 0.21 & 0.20 & 0.10 & 0.10 \\
\hline 9 & & & 0.62 & 0.58 & 0.54 & 0.39 \\
\hline 10 & & & & 0.12 & 0.11 & 0.11 \\
\hline 11 & & & & & 0.16 & 0.15 \\
\hline 12 & & & & & & 0.18 \\
\hline Mean interval & 7.00 & 7.31 & 8.44 & 8.72 & 9.14 & 9.67 \\
\hline Log-likelihood & -inf & -1004.6 & -974.9 & -973.5 & -971.6 & -970.9 \\
\hline verdict & reject & reject & just reject & just reject & select & \\
\hline
\end{tabular}

The results for the maximum age at first calving are not as clear-cut as the results for the maximum calving interval, because the number of known-age animals that have themselves been observed to calve was still very few in 1990. The mean age at first calving, assuming a maximum age at first calving of 11 years, is 9.1 years $(\mathrm{SE}=0.3$ years $)$. It is possible that this is still an underestimate and that more data might indicate yet higher ages at first calving. The estimated modal age at first calving is nine years, with about $50 \%$ of first calvings estimated to occur at this age (Fig. 4).

The corresponding survival probability of female calves to their first calving, which would be required to account for the increase rate of the population in the absence of immigration, is estimated using formula (18) to be 0.92 $(\mathrm{SE}=0.11)$. This implies an average annual mortality rate of calves and juveniles of about $0.01(\mathrm{SE}=0.01)$.

\section{DISCUSSION}

The results of this paper demonstrate that precise estimates of important demographic parameters, including the survival/mortality rate, the rate of population increase and the mean calving rate or calving interval, can be obtained from individual recognition data of this kind, provided that the data are collected over a long enough period.
Cataloguing and matching of the photographs obtained since 1990 should enable refinement of the estimate of the mean age at first calving and the opportunity to test whether the demographic rates are changing over time. As the data series lengthens, the labour involved in cataloguing new photographs by hand increases substantially, because each new photograph has to be checked against a larger catalogue. However, recent computer-based methods to aid matching (Hiby, 1999; Burnell et al., 2001) promise to considerably ease the task.

The estimates of biological parameters for this population obtained in this analysis are similar to but more precise than those obtained for the same population by Payne et al. (1990). This is partly due to the longer data series (1971-90 as opposed to 1971-86 in the earlier analysis), and partly due to the additional assumption in this analysis of a simple exponential trend in the expected numbers of calvings. The estimates of demographic parameters obtained for this population are similar to those obtained by Best et al. (2001) for the southeast Atlantic population of this species using virtually the same methods.

The sensitivity of our demographic parameter estimates to possible immigration or emigration is of interest in the light of evidence of possible interchange between populations (Best et al., 1993). In terms of the model used, permanent emigration is indistinguishable from mortality; if it occurs, the rate is subsumed into the estimated mortality rate. Temporary emigration spanning a calving, if it occurs, is indistinguishable from missed calvings; it will be reflected in the estimates of the recording probability but not in the demographic parameters. Temporary emigration between calvings has no effect on the data or estimates.

Since the model makes no assumptions about the extent of immigration, if any, estimates of demographic parameters remain valid in the presence of immigration. The estimate of the survival rate of female calves to first reproduction that would be required to account for the population increase rate in the absence of immigration, is high but not implausible $(0.92, \mathrm{SE}=0.11)$. Hence, the analysis provides no evidence that significant immigration has occurred.

\section{ACKNOWLEDGEMENTS}

We gratefully acknowledge the help of graduate students, researchers and friends during all phases of data collection and analysis. Particular thanks go to the pilots of the Aero Club of Trelew, photographers and their assistants who spent long and difficult hours collecting aerial survey data. Fred Adler (University of Utah), Lex Hiby (Conservation Research Ltd) and Les Underhill (University of Cape Town) provided valuable comments on the manuscript. We thank Estancia La Adela for use of their landing strip, A. Torrejon and N. Garcia of the Organismo Provincial de Turismo for permits to conduct the research, and the Wildlife Conservation Society for use of the research station. We are especially grateful to Aerolineas Argentinas, Fundacion Alfredo Fortabat, Sarah Haney, Minolta Inc., and the National Geographic Society, the People's Trust for Endangered Species, the Wildlife Conservation Society and the World Wildlife Fund for contributions that made this research possible.

\section{REFERENCES}

Barlow, J. and Clapham, P.J. 1997. A new birth-interval approach to estimating demographic parameters of humpback whales. Ecology 78(2):535-46 
Best, P.B., Payne, R., Rowntree, V., Palazzo, J.T. and Both, M.D. 1993. Long-range movements of South Atlantic right whales Eubalaena australis. Mar. Mammal Sci. 9(3):227-34.

Best, P.B., Brandão, A. and Butterworth, D. 2001. Demographic parameters of southern right whales off South Africa. J. Cetacean Res. Manage. (special issue) 2:161-169.

Burnell, S.R., and Shanahan, D. 2001. A note on a prototype system for simple computer-assisted matching of individually identified southern right whales (Eubalaena australis). J. Cetacean Res. Manage. (special issue) 2:297-300.

Cormack, R.M. 1981. Loglinear models for capture-recapture experiments on open populations. pp. 217-55. In: R.M. Cormack, G.P. Patil and D.S. Robson (eds.) Sampling Biological Populations. International Co-operative Publishing House, Fairland.

Hiby, L. 1999. Comparing patterns of detection to estimate abundance. Paper SC/M99/SOWER23 presented to the IWC Scientific Committee SOWER 2000 Workshop, March 1999. (unpublished). [Available from the Office of this Journal].
Payne, R. 1986. Long term behavioral studies of the southern right whale (Eubalaena australis). Rep. int. Whal. Commn (special issue) 10:161-7.

Payne, R. and Dorsey, E.M. 1983. Sexual dimorphism and aggressive use of callosities in right whales (Eubalaena australis). p. 643. In: R. Payne (ed.) Communication and Behavior of Whales. AAAS Selected Symposia 76. Westview Press, Colorado. xii+643pp.

Payne, R., Brazier, O., Dorsey, E.M., Perkins, J.S., Rowntree, V.J. and Titus, A. 1983. External features in southern right whales (Eubalaena australis) and their use in identifying individuals. pp. 371-445. In: R. Payne (ed.) Communication and Behavior of Whales. AAAS Selected Symposia 76. Westview Press, Colorado. xii+643pp.

Payne, R., Rowntree, V., Perkins, J.S., Cooke, J.G. and Lankester, K. 1990. Population size, trends and reproductive parameters of right whales (Eubalaena australis) off Peninsula Valdes, Argentina. Rep. int. Whal. Commn (special issue) 12:271-8. 